\title{
Post-exposure prophylaxis or pre-emptive therapy for severe acute respiratory syndrome coronavirus 2 (SARS-CoV-2): study protocol for a pragmatic randomized-controlled trial Prophylaxie post-exposition ou traitement préventif pour le syndrome respiratoire aigu sévère du coronavirus 2 (SARS- CoV-2) : protocole d'étude pour une étude randomisée contrôlée pragmatique
}

Sylvain A. Lother, MD - Mahsa Abassi, DO • Alyssa Agostinis, BSc $\cdot$ Ananta S. Bangdiwala, MSc Matthew P. Cheng, MDCM • Glen Drobot, MD $\cdot$ Nicole Engen, MSc $\cdot$ Kathy H. Hullsiek, PhD $\cdot$ Lauren E. Kelly , PhD • Todd C. Lee, MD, MPH, FIDSA · Sarah M. Lofgren, MD • Lauren J. MacKenzie, MD, MPH • Nicole Marten , RN • Emily G. McDonald, MD, MSc • Elizabeth C. Okafor, BS • Katelyn A. Pastick, BS • Matthew F. Pullen, MD • Radha Rajasingham, MD - Ilan Schwartz, MD, PhD - Caleb P. Skipper, MD - Alexis F. Turgeon, MD, MSc • Ryan Zarychanski, MD, MSc $\cdot$ David R. Boulware, MD, MPH

Received: 21 April 2020/ Accepted: 21 April 2020/Published online: 7 May 2020

(C) Canadian Anesthesiologists' Society 2020

\begin{abstract}
Background The severe acute respiratory syndrome coronavirus 2 (SARS-CoV-2) emerged in December 2019 causing the coronavirus disease (COVID-19) pandemic. Currently, there is a lack of evidence-based therapies to prevent COVID-19 following exposure to the virus, or to prevent worsening of symptoms following confirmed infection. We describe the design of a clinical trial of
\end{abstract}

S. A. Lother, MD (ه)

Department of Internal Medicine, Section of Critical Care,

University of Manitoba, Winnipeg, MB, Canada

e-mail: slother@manitoba-physicians.ca

Section of Infectious Diseases, Department of Internal Medicine, University of Manitoba, Winnipeg, MB, Canada

M. Abassi, DO - S. M. Lofgren, MD - E. C. Okafor, BS ·

K. A. Pastick, BS - M. F. Pullen, MD - R. Rajasingham, MD .

C. P. Skipper, MD - D. R. Boulware, MD, MPH

Department of Medicine, University of Minnesota, Minneapolis,

MN, USA

\section{A. Agostinis, BSc}

Division of Infectious Diseases, Department of Medicine, Faculty of Medicine and Dentistry, University of Alberta,

Edmonton, AB, Canada

School of Medicine, Trinity College Dublin, Dublin, Ireland hydroxychloroquine for post-exposure prophylaxis (PEP) and pre-emptive therapy (PET) for COVID-19.

Methods We will conduct two nested multicentre international double-blind randomized placebo-controlled clinical trials of hydroxychloroquine for: 1) PEP of asymptomatic household contacts or healthcare workers exposed to COVID-19 within the past four days, and 2) PET for symptomatic outpatients with COVID-19 showing symptoms for less than four days. We will recruit 1,500

A. S. Bangdiwala, MSc $\cdot$ N. Engen, MSc $\cdot$ K. H. Hullsiek, PhD Division of Biostatistics, School of Public Health, University of Minnesota, Minneapolis, MN, USA

M. P. Cheng, MDCM

Divisions of Infectious Diseases \& Medical Microbiology, McGill University Health Centre, Montreal, QC, Canada

McGill Interdisciplinary Initiative in Infection and Immunity, Montreal, QC, Canada

G. Drobot, MD

Department of Medicine, University of Manitoba, Winnipeg, MB, Canada

L. E. Kelly, PhD

Department of Pediatrics and Child Health, Department of Pharmacology, University of Manitoba, Winnipeg, MB, Canada

T. C. Lee, MD, MPH, FIDSA - E. G. McDonald, MD, MSc 
patients each for the PEP and PET trials. Participants will be randomized $1: 1$ to receive five days of hydroxychloroquine or placebo. The primary PEP trial outcome will be the incidence of symptomatic COVID-19. The primary PET trial outcome will be an ordinal scale of disease severity (not hospitalized, hospitalized without intensive care, hospitalization with intensive care, or death). Participant screening, informed consent, and follow-up will be exclusively internet-based with appropriate regulatory and research ethics board approvals in Canada and the United States.

Discussion These complementary randomized-controlled trials are innovatively designed and adequately powered to rapidly answer urgent questions regarding the effectiveness of hydroxychloroquine to reduce virus transmission and disease severity of COVID-19 during a pandemic. In-person participant follow-up will not be conducted to facilitate social distancing strategies and reduce risks of exposure to study personnel. Innovative trial approaches are needed to urgently assess therapeutic options to mitigate the global impact of this pandemic.

Trials registration clinicaltrials.gov (NCT04308668); registered 16 March, 2020.

Keywords Hydroxychloroquine - COVID-19 . clinical trials $\cdot$ coronavirus $\cdot \mathrm{SARS}-\mathrm{CoV}-2$. post-exposure prophylaxis · pre-emptive therapy · healthcare worker

Coronavirus disease (COVID-19) is a novel infection caused by severe acute respiratory syndrome-related coronavirus-2 (SARS-CoV-2). ${ }^{1}$ The virus was first recognized in Wuhan, China in December 2019 and

Clinical Practice Assessment Unit, Department of Medicine, McGill University, Montreal, QC, Canada

L. J. MacKenzie, MD, MPH

Section of Infectious Diseases, Department of Internal Medicine, University of Manitoba, Winnipeg, MB, Canada

Department of Community Health Sciences, University of Manitoba, Winnipeg, MB, Canada

N. Marten, RN

Critical Care Research, St-Boniface Hospital, Winnipeg, MB, Canada

I. Schwartz, MD, $\mathrm{PhD}$

Division of Infectious Diseases, Department of Medicine, Faculty of Medicine and Dentistry, University of Alberta, Edmonton, AB, Canada quickly spread globally, leading to a pandemic. $^{2}$ Currently, the standard of care in most jurisdictions for those with mild to moderate COVID-19 includes selfobservation and self-quarantine for seven to 14 days. ${ }^{3,4}$ While some small studies ${ }^{5-8}$ with methodologic limitations have suggested certain therapies may have promise, at the time of this publication, no proven effective therapies exist. Most major national and international guidelines have yet to incorporate pharmacologic therapies for COVID-19 because high-grade evidence is lacking. ${ }^{9,10}$ An effective post-exposure prophylaxis (PEP) or pre-emptive therapeutic (PET) intervention would provide significant benefit to individuals, as well as to public health, given the high rate of SARS-CoV-2 transmission and overburdened healthcare systems.

Coronavirus disease 2019 symptoms generally develop an average of four to five days after exposure to SARSCoV-2. ${ }^{11}$ The incubation period ranges from one to 14 days. $^{12}$ The majority (80\%) of people with COVID-19 exhibit mild symptoms and recover spontaneously. ${ }^{13}$ Nevertheless, $14 \%$ of patients develop hypoxemia requiring hospitalization, admission to the intensive care unit (ICU), and/or invasive ventilation. ${ }^{14}$ Of those who are hospitalized in North America, approximately 30\% of individuals will develop respiratory failure, shock, and/or multiple organ system dysfunction requiring admission to a critical care unit. $^{15}$

Severe acute respiratory syndrome-related coronavirus-2 has spread rapidly through the population with a basic reproductive value $\left(\mathrm{R}_{0}\right)$ value of 2 to $5.7 .^{16,17}$ The secondary attack rate among household contacts has been estimated at $10 \%$ (95\% confidence interval [CI] 2 to 31$),{ }^{18}$ but estimates are largely based on monitoring of travelassociated COVID-19 cases. In the context of community transmission, the secondary attack rate may be as high as $35 \%$ (95\% CI 27 to 44$).{ }^{19}$ In the absence of appropriate

A. F. Turgeon, MD, MSc

CHU de Québec - Université Laval Research Centre, Population Health and Optimal Health Practices Research Unit Trauma -

Emergency - Critical Care Medicine, Université Laval, Quebec, QC, Canada

Department of Anesthesiology and Critical Care Medicine, Division of Critical Care Medicine, Faculty of Medicine, Université Laval, Quebec, QC, Canada

R. Zarychanski, MD, MSc

Department of Internal Medicine, Section of Critical Care, University of Manitoba, Winnipeg, MB, Canada

Department of Internal Medicine, Section of Hematology and Oncology, University of Manitoba, Winnipeg, MB, Canada

Department of Medical Oncology and Hematology, CancerCare Manitoba, Winnipeg, MB, Canada 
distancing strategies, secondary attack rates may be significantly higher. Since up to $14 \%$ of patients with secondary infections will require hospitalization and are at risk of poor outcomes, including death, a protective therapeutic strategy is urgently needed following exposure to COVID-19.

While few pharmacologic agents have shown in vitro suppression of viral replication against coronaviruses, ${ }^{20}$ one agent, chloroquine, has been shown to be effective at reducing laboratory established infection and spread to adjacent cells from SARS-CoV. ${ }^{21}$ More recently, the related drug hydroxychloroquine was found to prevent SARS-CoV-2 viral replication before and after infection of Vero cell lines. ${ }^{22}$ The exact antiviral mechanism of hydroxychloroquine against SARS-CoV-2 is incompletely understood, but may be related to impaired angiotensin converting enzyme- 2 receptor binding and resultant cell entry, as well as a suppressed "cytokine storm". ${ }^{23}$

Recent news outlet reports of chloroquine poisoning have raised concerns regarding the safety of hydroxychloroquine, but scientific details are lacking. A recent trial of chloroquine among patients hospitalized with COVID-19 in Brazil was stopped early $(n=81)$ because of safety concerns. Patients receiving high-dose chloroquine (12 $\mathrm{g}$ over ten days) had prolonged QTc (> $500 \mathrm{msec}$ ) in $25 \%$ of cases, including two deaths secondary to ventricular tachycardia. ${ }^{24}$ This dose was orders of magnitude higher than for malaria prophylaxis $(500 \mathrm{mg}$ weekly) or malaria treatment (2.5 g total), each of which have well-established safety profiles. $^{25}$ Hydroxychloroquine is much less toxic that chloroquine, ${ }^{26}$ and has decades of established safety data in the treatment of malaria and other autoimmune conditions. $^{27}$ Preliminary data in COVID-19 trials have shown increased gastrointestinal upset but no significant differences in serious adverse events. ${ }^{28}$

Early translational and clinical studies have shown that hydroxychloroquine might be an effective pharmacologic treatment to prevent viral establishment if used early after exposure or established infection. ${ }^{5-8,29}$ Delaying treatment to the time of hospitalization, which occurs on average seven to eight days from symptom onset, ${ }^{30,31}$ may be associated with a lack of benefit because of established viral infection. ${ }^{28,32}$ Nevertheless, the current evidence base is limited because of small, underpowered sample sizes and large observational studies without proper controls. ${ }^{20}$ Global political forces continue to emphasize a not-yetproven role for hydroxychloroquine in the paradigm of COVID-19 treatment. Urgent clinical trials are therefore needed to establish whether hydroxychloroquine may be an effective therapy for COVID-19.

\section{Trial objectives}

To determine if hydroxychloroquine compared with placebo is effective at: 1) preventing COVID-19 disease after exposure to a known case (i.e., PEP), and 2) preventing severe complications of the disease if started early in the course of illness (i.e., PET).

\section{Trial design}

This trial is a double-blind placebo-controlled multicentre international randomized-controlled trial designed to evaluate the superiority of hydroxychloroquine vs placebo. Participants will be enrolled in two nested trials that will be randomized separately and have separate endpoints but use the same workflow, study team, documents, and infrastructure. The PEP trial will include asymptomatic healthcare workers or household contacts exposed to COVID-19-positive cases within four days. The PET trial will include outpatients with COVID-19 and showing symptoms for four or fewer days. Each trial is planned to recruit a total of 1,500 patients. Within each trial, participants will be randomized 1:1 in parallel groups to receive hydroxychloroquine or placebo. This clinical trial was registered at Clinical Trials.gov (NCT04308668) on 16 March 2020 and was approved by the Food and Drug Administration (FDA; IND 148257) and Health Canada. Research ethics board (REB) approval was obtained at the University of Minnesota (16 March 2020), McGill University Health Centre Research Institute (1 April 2020), University of Alberta (6 April 2020), and University of Manitoba (25 March 2020), prior to recruitment of the first participant.

\section{Methods}

Trial setting

Participants will be non-hospitalized adults recruited from the community via advertisements and using traditional methods and social media (Twitter, Instagram, Facebook). Recruitment will take place throughout the United States (US), and, at least initially, within three provinces in Canada (Quebec, Manitoba, Alberta). Approval is being sought in additional provinces. To limit the spread of disease, no in-person visits with study personnel will be performed. During recruitment and follow-up, most participants will be at home in self-isolation or quarantine; screening, informed consent, randomization, and data collection will occur online through the Research Electronic Data Capture (REDCap), a secure web-based 
platform. Study medications will be delivered directly to the participants' place of residence via courier services that respect safety protocols for home deliveries to patients with COVID-19. Participants can contact the local study teams in their region via email or phone for questions or concerns with timely responses (same day during business hours and the following day after $9 \mathrm{pm}$ ). Websites have been created that will provide live enrolment updates, responses to frequently asked questions, and trial information for participants (www.covidpep.umn.edu and www.covid19research.ca).

Inclusion criteria

- Adults ( $\geq 18$ yr old)

- Able to read and comprehend French or English

- Access to the internet with valid email address for enrolment and follow-up surveys.

Post-exposure prophylaxis

1) At-risk exposure to a COVID-19-positive case within the past 4 days

2) Household contact or healthcare worker

Pre-emptive therapy

1) Non-hospitalized community-dwelling symptomatic (fever, cough, or shortness of breath) COVID-19 within four days of symptom onset

2) Coronavirus disease confirmed by reverse transcriptase polymerase chain reaction (RT-PCR) or for healthcare workers, by compatible symptoms following exposure to a person with a known positive RT-PCR result (exposure occurring within the past 14 days).

Enrolment for the PEP trial will be limited to asymptomatic individuals exposed to a COVID-19confirmed case within $\leq$ four days, as the mean incubation period for SARS-CoV-2 is five days. ${ }^{12}$ Household contacts are defined as residing in the same domicile. At-risk exposures in the healthcare setting are defined as greater than ten minutes spent within $2 \mathrm{~m}$ of a COVID-19-confirmed case, with insufficient use of personal protective equipment per institutional guidelines. Healthcare workers are self-identified physicians, nurses, respiratory therapists, or other workers providing direct patient contact. This will select a high-risk group for developing clinical infection but within the time period that the intervention could prevent or ameliorate disease. All efforts will be made to deliver study medicines before the fourth day following exposure; however, delays may lead to symptom onset after randomization but prior to starting the intervention. These events will be tracked by online surveys administered upon receipt of the intervention. Because community testing for COVID-19 is limited, participants will be included in the PET group as long as symptoms are compatible with COVID-19 after exposure to a person with confirmed COVID-19.

\section{Exclusion criteria}

1) Known allergy to chloroquine or hydroxychloroquine

2) Current use of chloroquine or hydroxychloroquine for any reason

3) Currently hospitalized

4) Known chronic kidney disease, stage $4-5$, or dialysis $^{33}$

5) Active malignancy on systemic chemotherapy

6) Known porphyria

7) Prior retinal eye disease

8) Known glucose-6-phosphate dehydrogenase (G6PD) deficiency

9) Weight $<40 \mathrm{~kg}$

10) Known history of ventricular arrhythmia, prolonged QTc interval (personal or family history), structural or ischemic heart disease, or any known episode of sudden cardiac death

11) Severe diarrhea and/or vomiting at screening that may interfere with drug absorption

12) Significant hepatic impairment defined as known cirrhosis with a history of hepatic encephalopathy or ascites

13) Known pregnancy or breastfeeding

14) Current use of any of the following medications with known drug-drug interactions: amiodarone, amitriptyline, artemether, azithromycin, ciprofloxacin, citalopram, clarithromycin, dapsone, desipramine, digoxin, dofetilide, doxepin, droperidol, erythromycin, escitalopram, flecainide, fluoxetine, haloperidol, imipramine, itraconazole, ketoconazole, levofloxacin, lithium, lumefantrine, mefloquine, methadone, moxifloxacin, procainamide, propafenone, quetiapine, sertraline, sotalol, sumatriptan, thioridazine, venlafaxine, bupropion, ziprasidone, zolmitriptan

15) Current use of any anti-epileptic.

Exclusion criteria 11-14 are specific to participants in Canada at the request of Health Canada. Exclusion criteria 15 is specific to participants in the US. In the US, the FDA has approved the inclusion of pregnant and breastfeeding women citing hydroxychloroquine safety data in pregnant 
Figure Participant schedule of enrolment, interventions, and assessments. *Follow-up out to day 90 if participants remain hospitalized.

\begin{tabular}{|c|c|c|c|c|c|c|c|c|}
\hline & \multicolumn{8}{|c|}{ Study Period } \\
\hline & \multirow{2}{*}{$\begin{array}{c}\text { Enrolment } \\
\text { D0 }\end{array}$} & \multirow{2}{*}{$\begin{array}{c}\text { Allocation } \\
\text { D0 }\end{array}$} & \multicolumn{4}{|c|}{ Post-allocation } & \multicolumn{2}{|c|}{ Close-out } \\
\hline Time Points & & & D1 & D3 & D5 & D9 & D14 & D90 \\
\hline \multicolumn{9}{|l|}{ ENROLMENT } \\
\hline Online eligibility screen & $\mathrm{X}$ & & & & & & & \\
\hline Online informed consent & $\mathrm{X}$ & & & & & & & \\
\hline Allocation & & $\mathrm{X}$ & & & & & & \\
\hline Drug couriered & & $\mathrm{X}$ & & & & & & \\
\hline \multicolumn{9}{|l|}{ INTERVENTIONS } \\
\hline \multicolumn{9}{|l|}{ Hydroxychloroquine } \\
\hline \multicolumn{9}{|l|}{ Placebo } \\
\hline \multicolumn{9}{|l|}{ ASSESSMENTS } \\
\hline Online survey & & & $\mathrm{X}$ & & $\mathrm{X}$ & & $\mathrm{X}$ & \\
\hline $\begin{array}{l}\text { Additional online surveys } \\
\text { (if symptomatic) }\end{array}$ & & & & $\mathrm{X}$ & & $\mathrm{X}$ & & $\mathrm{X}^{*}$ \\
\hline
\end{tabular}

The rationale for the study dose is adapted from a modified malaria dosing schedule for

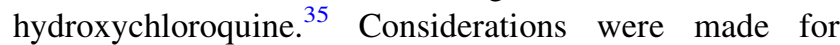
maximal viral inhibition while balancing potential toxicity and drug supply with anticipated shortages. A loading dose was used, similar to malaria treatment strategy to achieve antiviral activity as quickly as possible. Monte Carlo simulations were conducted with the proposed dose achieving steady-state serum concentrations above the EC50, ${ }^{22}$ where $50 \%$ of viral inhibition would occur.

The most common anticipated side effect from hydroxychloroquine is gastrointestinal upset. $^{27}$ Participants will be instructed to spread out doses throughout the day (e.g., two tablets with breakfast, one tablet with lunch) to increase tolerability if required. Participants may voluntarily stop taking the study medicine at any time. Adherence will be determined by self-report. Chronic medications will be taken as usual, but these must be taken 4 hours before or after taking the study medicine. No other concomitant COVID-19 therapy or other trial medicines are permitted for the duration of the trial. In the event of hospitalization, unblinding of group assignment can occur at the request of the treating physician, and participants may receive other medications or therapies, including experimental treatments from other trials.
Placebo tablets will be administered using the same dosing schedule and number of tablets as the interventional arm. 


\section{Outcomes}

\section{Primary OUTCOME FOR PEP}

1) Incidence of proven COVID-19 disease onset by 14 days.

In the absence of SARS-CoV-2 testing, COVID-19 diagnosis will be made using clinical criteria and labelled as a possible case. For the "confirmed cases" outcome, there may be limitations related to access to testing. We will use an a priori clinical definition using the symptoms most commonly reported in PCR-proven patients from the World Health Organization (WHO). ${ }^{36}$

\section{PRIMARY OUTCOME FOR PET}

1) Ordinal scale for clinical severity, defined by WHO COVID-19 therapeutic trial synopsis ${ }^{37}$

$$
\begin{aligned}
& 1=\text { Ambulatory } \\
& 2=\text { Hospitalized with mild disease } \\
& 3=\text { Hospitalized with severe disease } \\
& 4=\text { Death (all-cause). } \\
& \text { SECONDARY OUTCOMES FOR PEP AND PET }
\end{aligned}
$$

1) Incidence of hospitalization for COVID-19;

2) Incidence of death from COVID-19;

3) Incidence of confirmed SARS-CoV-2 detection;

4) Incidence of possible COVID-19 symptoms;

5) Incidence of all-cause study medicine discontinuation;

6) Severity of symptoms at day 5 and 14 by visual analogue scale.

The primary and secondary outcomes listed will be analyzed separately for both trials. Outcome measures will be assessed through self-report via online questionnaires. Wherever possible, COVID-19 disease status will be verified from public health records, medical records, or death certificates. Patients with positive RT-PCR results for SARS-CoV-2 in a nasopharyngeal swab will be considered confirmed cases. ${ }^{38}$ rT-PCR will be performed as per the local institution's standard procedures.

For patients with COVID-19 who require hospitalization, outcome data will be collected up to day 90. Participants will complete additional online surveys throughout hospitalization to assess for adverse events related to the study medicine and their COVID-19-related outcomes. All participants will complete virtual assessments on days 1, 5, and 14 (Figure). If symptomatic disease develops, participants will be required to complete additional virtual assessments on days 3 and 10. Participants who are pregnant or become pregnant while participating in the trial will be followed to the post-partum period to assess maternal and fetal outcomes. In the event that a participant does not respond to the day 14 survey, we will contact them by telephone, by registered mail, and if they are still not available, all participants have given a third-party telephone contact to verify vital status.

\section{Recruitment and consent}

Recruitment and follow-up will be exclusively internetbased. Prospective participants will visit the trial website, read the provided study information, and can click the link to enrol. The trial website will be advertised through communication strategies and media channels, and through internet-based advertisements and social media. In all regions, COVID-19-positive cases are reported to public health officials where positive patients are notified, and contact tracing is initiated. In some jurisdictions, public health officials will provide information on accessing the trial website. Healthcare workers will be notified of the trial website by email communications and through the offices of occupational health and promotion by senior hospital administration. Online screening questionnaires will assess interested participants for eligibility criteria. Eligible participants will provide informed consent by submitting an online form, linked through the trial website. Contact information will be provided for participants to ask questions by email and/or phone.

Randomization sequence generation, allocation concealment, and blinding

Participants will be randomized via computer-generated permuted block randomization and stratified into the PEP trial (for asymptomatic participants) or the PET trial (for symptomatic participants). The randomization sequence will be pre-specified. Randomization will be recorded on an electronic log, which will only be accessed by the research pharmacy, those packaging study medication, and the unblinded statisticians. Study investigators, trial participants, care providers, outcome assessors, and data analysts will be blinded to the allocation sequence. Once study medication is prepared by the onsite pharmacies, packages will be shipped directly to participants via courier.

Participants will be provided masked study medicine and will not be notified whether they are taking hydroxychloroquine or placebo. Instructions for taking the medicine are identical between the groups. Because a variety of sources of hydroxychloroquine are being used in different jurisdictions, the placebo will not be identically matched to the hydroxychloroquine tablet; however, both placebo and hydroxychloroquine will have similar rounded oval shapes, colour, and indistinct markings making unblinding of the trial arm unlikely. Patients will be asked if they believe they received the intervention or 
control upon trial closure. In the event of a medical emergency, an on-call investigator will be available for code breaking if required. Any code breaking event will be reported to the research pharmacy and principal investigator.

Data collection methods and data management

All data collection, including baseline demographics, clinical and epidemiological characteristics, study medicine tolerability, adherence, and outcomes will be recorded online through self-report captured in internetbased questionnaires (administered in REDCap). All participant-submitted data will be stored and maintained in a secure server. Data from the US will be stored on the secure servers at the University of Minnesota, and data from Canada will be stored on the secure servers at the Research Institute of the McGill University Health Centre. Both the US and Canadian databases will be stored and managed in their respective countries. For interim data safety monitoring board purposes and also upon completion of recruitment, confidential de-identified data will be shared to facilitate analysis through institutional data sharing agreements. No paper documents will be retained or stored.

Enrolment progress reports will be generated for each trial at $25 \%, 50 \%$, and $75 \%$ of enrolment to highlight the number of participants enrolled, on study, completed, or lost to follow-up. The cumulative incidence of COVID-19 (both arms pooled) and cumulative hospitalizations (both arms pooled) will be reported.

\section{Sample size}

According to existing research, the attack rate of transmission to household contacts is approximately $10 \% .{ }^{18}$ We used Fisher's exact test and a two-sided $\alpha=$ 0.05 and test power of $\beta=0.90$ to detect a $50 \%$ relative risk reduction in disease incidence. Using 1:1 randomization, we calculated a required sample size of $n=621$ per arm. Accounting for a predicted $20 \%$ dropout rate with an internet self-study design the sample size was inflated to $\mathrm{n}$ $=746$ per arm. Therefore, we aimed to recruit a total of 1,500 patients for the PEP trial. For symptomatic patients, the proportion of patients who progress to require hospitalization is approximately $10 \% .{ }^{14}$ For this trial, we assumed that without treatment, $90 \%$ of the control arm would not be hospitalized, $8 \%$ would be hospitalized without an ICU stay or death, and $2 \%$ would be hospitalized with an ICU stay or death. With 1:1 randomization and 1,464 total participants, the PET trial has $90 \%$ power to detect a log odds ratio of 0.70 . A number of sample size approximations were made accounting for differences in baseline rates and relative effect sizes. These can be found in Appendices 1 and 2.

Statistical methods

Primary and secondary outcomes will be analyzed separately in PEP and PET trials by intention-to-treat.

\section{PRIMARY OUTCOME FOR PEP}

The incidence of COVID-19 by day 14 will be assessed in the intervention $v s$ control arm by the Fisher's exact test.

PRIMARY OUTCOME FOR PET

The ordinal scale for disease severity (outpatient, hospitalized without ICU, hospitalized with ICU, death) at day 14, will be assessed in the intervention $v s$ control arms by proportional odds model.

Secondary outcomes (PEP and PET aRe ANALYZEd SEPARATELY)

In the PEP group, for the subset of patients who develop symptoms of COVID-19 after taking at least one dose of the study medicine, we will analyze the change in symptom severity in the intervention $v s$ control group. We will use the same approach as for the primary outcome in the PET group. Participants who are randomized in the PEP group but develop symptoms before taking one dose of the study medicine will be described and analyzed separately in a subgroup analysis.

Secondary outcomes for incidence will be reported with the Fisher's exact test. Continuous valued secondary outcomes will be reported by mean (standard deviation) or median [interquartile range], depending on their normality. Analysis will be conducted by parametric or non-parametric tests as appropriate. Symptom severity scores will be recorded on days $0,1,3,5,10$, and 14 using a $10-\mathrm{cm}$ continuous visual analogue scale, where participants can electronically control a slider indicating symptom severity, with $0=$ no symptoms and $10=$ severe symptoms. Measurements will be recorded to the nearest $0.1 \mathrm{~cm}$. The severity of symptoms on day 5 will be compared first by categorical analysis (symptoms present yes or no) via Fisher's exact Chi square, and subsequently via the independent two-sample $t$ test for symptom severity among those who are symptomatic. Non-normally distributed data will be analyzed via the Mann-Whitney U test.

Preplanned subgroup analyses

1) Participants contact had SARS-CoV-2 confirmed by positive molecular test result;

2) Participant had SARS-CoV-2 confirmed by positive molecular test result;

3) Exposure in healthcare worker vs household contact; 
4) Number of days from the exposure;

5) Decile of age;

6) Sex as a biological variable;

7) Censored subjects in the PEP trial who become symptomatic before taking one dose of the study medicine.

\section{Data monitoring}

A Data Safety Monitoring Board (DSMB) will be formed independent of the sponsor and without competing interests. For each trial (PEP and PET), an interim analysis will be presented to the DSMB once the 14-day follow-up is completed for $25 \%, 50 \%$, and $75 \%$ of enrolled participants. All interim analyses will pool US and Canadian de-identified data to arrive at a generalizable conclusion as early as possible. Only the DSMB and unblinded statisticians will be provided with unblinded interim analysis results. The investigators will have access to only pooled results. A Lan-DeMets spending function analogue of the O'Brien-Fleming boundaries for the primary outcome for each group will be provided to the DSMB. The stopping boundaries will be truncated at $\alpha=$ 0.001 ( $|Z|>3.09)$. At each analysis, data will be reviewed for safety/efficacy and for achieving stopping rules. Should stopping rules for the primary outcome be met, the DSMB will review secondary outcomes for consistency such that a clear answer is achieved. In the event of early termination for efficacy, the trial will immediately convert to an openlabel observational cohort study.

At the second DSMB review, a sample size reestimation will occur based on the disease transmission rate in the control group. The a priori assumption of $10 \%$ transmission risk in close contacts is based on limited data in other jurisdictions. A new sample size estimation will take into account the updated transmission rate in this trial and be powered to detect a $50 \%$ relative reduction in the primary outcome. Starting with the second DSMB review, the DSMB will be given the conditional power under both the trial design parameters and under the current data. If the conditional power is less than $20 \%$, trial discontinuation may be considered.

\section{Potential harms}

Short-term use of hydroxychloroquine is well-tolerated with a safe track record of use for approved indications since 1955 . The most commonly reported side effects include: gastrointestinal upset, nausea, vomiting, diarrhea, headache, skin rash, or itching. ${ }^{27}$ Gastrointestinal side effects are minimized when taken with meals and when dosages are separated throughout the day. Participants will be instructed to use these optional strategies to increase tolerance.

While a recent study showed a risk of harm with very high doses of chloroquine ${ }^{24}$ hydroxychloroquine is much less toxic. ${ }^{26}$ Doses used in this trial (3.8 g total) are similar to those doses used to treat malaria ( $2 \mathrm{~g}$ total), and are significantly less than doses used to treat Coxiella burnetii infection or other autoimmune conditions (400-600 mg daily, indefinitely), all showing a long history of safety. ${ }^{27}$ A recent trial in COVID-19 using significantly higher doses ( $1.2 \mathrm{~g}$ daily for three days, then $800 \mathrm{mg}$ daily for two to three weeks) showed no significant differences in serious adverse advents, though gastrointestinal side effects were higher at this dose. ${ }^{28}$

The safety of taking hydroxychloroquine will be enhanced by excluding those with pre-existing retinopathy, allergic reactions, cardiac conditions, and certain other medications. All adverse reactions will be documented via self-report during virtual visits. Participants will be provided with contact information to be guided on how to manage adverse effects. There are no predefined plans for post-trial care above the standard of care offered at each centre. Participants will be provided with updated public health information on the COVID-19 pandemic and instructions for how to proceed in the event of a medical emergency.

\section{Auditing}

Regulatory agencies, institutional sponsors, and REBs are authorized to conduct trial audits throughout.

\section{Confidentiality and access to data}

Interactions with trial participants will be through internetbased REDCap electronic case report forms, conforming to required privacy and server security standards. No participant identifying information will be disclosed in any publication or in any other activities arising from this trial. Anonymized data will be pooled with international collaborators, subject to inter-institutional agreements. No information concerning this trial will be released to unauthorized third parties without the prior written approval of the participants, except for monitoring by the REBs or public health authorities. Only immediate study personnel will be authorized to access the database.

Dissemination policy

Any protocol amendments will be reviewed with each REB and updated on the trial registry and websites. Trial enrolment and results, when available, will be updated on www.clinicaltrials.gov and will be publicly available on 
the trial websites (www.covidpep.umn.edu and www. covid-19research.ca). Because of the nature of this pandemic, pooled and anonymized trial results will be published immediately, once available, on open access websites and will be submitted urgently for accelerated peer reviewed publication. Full de-identified data will be made available to qualified researchers upon request.

\section{Discussion}

Hydroxychloroquine has garnered unprecedented attention as a potential therapeutic option in the global COVID-19 pandemic. ${ }^{39}$ Several in vitro and in vivo studies have shown data that may support its possible efficacy, but clinical data are limited to small clinical trials and uncontrolled case series and cohorts. ${ }^{20}$ There is an urgent need for the rigorous evaluation of hydroxychloroquine as a therapeutic option against COVID-19. ${ }^{40}$

This innovative trial is adequately powered to rapidly answer urgent questions regarding the effectiveness of hydroxychloroquine and its ability to prevent or reduce the severity of COVID-19. The novel web-based design of this trial, in the setting of a highly contagious global pandemic, has ensured that all interactions with participants, including enrolment and informed consent, are done remotely using courier services to deliver medicine and using internetbased follow-up. This will allow compliance with strict social distancing strategies and reduce risks of infectious spread to study personnel and others. The design encourages large scale enrolment to ensure the target number of participants are enrolled and trial results are obtained as quickly as possible. Importantly, the design was finalized, regulatory and REB approvals were obtained, and large geographic jurisdictions brought online in an unprecedently short duration (within a few weeks of a global pandemic) with a goal to obtain the maximal information in the minimal amount of time.

Recently, several public figures have promoted the widespread use of hydroxychloroquine in the absence of robust scientific data. ${ }^{39,41}$ This trial will help address if large scale dissemination of hydroxychloroquine is appropriate in certain populations. Importantly, if this study shows a positive effect, this will support a new therapeutic option to mitigate the global spread and impact of COVID-19. If a null or negative effect is shown, this will reduce the risk of harm to future patients, reduce healthcare expenditures due to ineffective medications, and protect the drug supply for patients with previously established indications for hydroxychloroquine. The results of this trial will be instrumental in determining if hydroxychloroquine may play an important role in mitigating the global impact of COVID-19.
Author contributions American sponsor: David R. Boulware; Canadian sponsor: Research Institute of the McGill University Health Centre; American principal investigator: David $R$. Boulware; Canadian principal investigators: Ryan Zarychanski, Todd C. Lee, Ilan Schwartz; Trial design: David R. Boulware, Todd C. Lee, Kathy H. Hullsiek; Research coordinators: Alyssa Agostinis, Nicole Marten, Zina Zaslawski; Data collection: Ananta Bangdiwala, Emily G. McDonald, Matthew P. Cheng, Sarah Lofgren, Matt Pullen, Caleb Skipper, Katelyn A. Pastick; Statistical analysis: Ananta Bangdiwala, Kathy Hullsiek, Nicole Engen; Operational support: Sylvain A. Lother, Lauren J. MacKenzie, Alyssa Agostinis, Mahsa Abassi, Katelyn A. Pastick, Elizabeth C. Okafor, Matt Pullen, Lauren E. Kelly, Ananta Bangdiwala, David R. Boulware; Protocol manuscript first draft: Sylvain A. Lother.

Disclosures Drs Emily G. McDonald and Todd C. Lee receive salary support from the Fonds de recherche du Québec-Santé. Dr. Cheng is a member of the scientific advisory board of GEn1E Lifesciences. Ryan Zarychanski is the recipient of the Lyonel G Israsels Professorship in Hematology at the University of Manitoba. Dr. Turgeon is the Canada Research Chair in Critical Care Neurology and Trauma. Additional authors declare no conflicts of interest.

Protocol Version 1.36, March 252020.

Funding statement U.S. Funding by Jan and David Baszucki, Alliance of Minnesota Chinese Organizations, Minnesota Chinese Chamber of Commerce, and University of Minnesota. Canadian funding by the Manitoba Medical Service Foundation. The funders will not contribute to the design of the study; collection, management, analysis, and interpretation of data; writing of the report; and the decision to submit the report for publication. Purolator Canada provided in-kind courier support for the participating Canadian sites.

Editorial responsibility This submission was handled by Dr. Gregory L. Bryson, Deputy Editor-in-Chief, Canadian Journal of Anesthesia.

\section{Appendix 1 Sample size calculation table for the post-} exposure prophylaxis study using Fisher's exact test

\begin{tabular}{|c|c|c|c|c|}
\hline $\begin{array}{l}\text { Estimated } \\
\text { transmission } \\
\text { rate with } \\
\text { placebo }\end{array}$ & $\begin{array}{l}\text { Estimated } \\
\text { transmission } \\
\text { rate with } \\
\text { drug }\end{array}$ & $\begin{array}{l}\text { Percent } \\
\text { relative } \\
\text { reduction in } \\
\text { transmission } \\
\text { rate }\end{array}$ & $\begin{array}{l}\text { Sample } \\
\text { size (per } \\
\text { arm) with } \\
90 \% \\
\text { power }\end{array}$ & $\begin{array}{l}\text { Sample } \\
\text { size (per } \\
\text { arm) with } \\
80 \% \\
\text { power }\end{array}$ \\
\hline \multirow[t]{2}{*}{$10 \%$} & $5 \%$ & $50 \%$ & 621 & 474 \\
\hline & $6 \%$ & $40 \%$ & 1014 & 771 \\
\hline \multirow[t]{2}{*}{$12 \%$} & $6 \%$ & $50 \%$ & 509 & 389 \\
\hline & $7.2 \%$ & $40 \%$ & 831 & 632 \\
\hline \multirow[t]{2}{*}{$15 \%$} & $7.5 \%$ & $50 \%$ & 398 & 304 \\
\hline & $9 \%$ & $40 \%$ & 648 & 493 \\
\hline
\end{tabular}


Appendix 2 Sample size calculation table for the preemptive therapy study using Fisher's exact test

\begin{tabular}{llll}
\hline $\begin{array}{l}\text { Log } \\
\text { odds } \\
\text { ratio }\end{array}$ & $\begin{array}{l}\text { Odds } \\
\text { ratio }\end{array}$ & $\begin{array}{l}\text { Sample size (per group) } \\
\text { with 90\% power }\end{array}$ & $\begin{array}{l}\text { Sample size (per group) } \\
\text { with 80\% power }\end{array}$ \\
\hline 0.60 & 1.82 & 805 & 601 \\
0.65 & 1.91 & 697 & 521 \\
0.70 & 2.01 & 610 & 456 \\
0.75 & 2.12 & 539 & 403 \\
\hline
\end{tabular}

\section{References}

1. Coronaviridae Study Group of the International Committee on Taxonomy of Viruses. The species severe acute respiratory syndrome-related coronavirus: classifying 2019-nCoV and naming it SARS-CoV-2. Nat Microbiol 2020; 5: 536-44.

2. World Health Organization. Report of the WHO-China Joint Mission on Coronavirus Disease 2019 (COVID-19); 2020. Available from URL: https://www.who.int/docs/default-source/ coronaviruse/who-china-joint-mission-on-covid-19-final-report. pdf (accessed April 2020).

3. Public Health Agency of Canada. Coronavirus disease (COVID19): Prevention and risks; 2020. Available from URL: https:// www.canada.ca/en/public-health/services/diseases/2019-novelcoronavirus-infection/prevention-risks.html (accessed April 2020)

4. Centers for Disease Control and Prevention. Coronavirus Disease 2019 (COVID-19) - Infection Control; 2020. Available from URL: https://www.cdc.gov/coronavirus/2019-ncov/hcp/ infection-control.html (accessed April 2020).

5. Chen J, Liu D, Liu L, et al. A pilot study of hydroxychloroquine in treatment of patients with common coronavirus disease-19 (COVID-19). J Zhejiang Univ (Med Sci) 2020; DOI: https://doi. org/10.3785/j.issn.1008-9292.2020.03.03.

6. Gao J, Tian Z, Yang X. Breakthrough: chloroquine phosphate has shown apparent efficacy in treatment of COVID-19 associated pneumonia in clinical studies. Biosci Trends 2020; 14: 72-3.

7. Gautret P, Lagier JC, Parola $P$, et al. Hydroxychloroquine and azithromycin as a treatment of COVID-19: results of an openlabel non-randomized clinical trial. Int $\mathrm{J}$ Antimicrob Agents 2020; DOI: https://doi.org/10.1016/j.ijantimicag.2020.105949.

8. Chen Z, Hu J, Zhang Z, et al. Efficacy of hydroxychloroquine in patients with COVID-19: results of a randomized clinical trial. medRxiv 2020; DOI: https://doi.org/10.1101/2020.03.22. 20040758 (accessed April 2020).

9. Alhazzani W, Moller MH, Arabi YM, et al. Surviving sepsis campaign - guidelines on the management of critically ill adults with coronavirus disease 2019 COVID-19. Crit Care Med 2020; DOI: https://doi.org/10.1097/CCM.0000000000004363.

10. Bhimraj A, Morgan RL, Shumaker AH, et al. Infectious Diseases Society of America Guidelines on the Treatment and Management of Patients with COVID-19; 2020. Available from
URL: https://www.idsociety.org/practice-guideline/covid-19guideline-treatment-and-management/ (accessed April 2020).

11. Linton NM, Kobayashi T, Yang Y, et al. Incubation period and other epidemiological characteristics of 2019 novel coronavirus infections with right truncation: a statistical analysis of publicly available case data. J Clin Med 2020; DOI: https://doi.org/10. 3390/jcm9020538.

12. Guan WJ, Ni ZY, Hu Y, et al. Clinical characteristics of coronavirus disease 2019 in China. N Engl J Med 2020; DOI: https://doi.org/10.1056/NEJMoa2002032.

13. Wu Z, McGoogan JM. Characteristics of and important lessons from the coronavirus disease 2019 (COVID-19) outbreak in China: summary of a report of 72314 cases from the Chinese Center for Disease Control and Prevention. JAMA 2020; DOI: https://doi.org/10.1001/jama.2020.2648.

14. Li $Q$, Guan $X, W u P$, et al. Early transmission dynamics in Wuhan, China, of novel coronavirus-infected pneumonia. N Engl J Med 2020; 382: 1199-207.

15. Bialek $S$, Boundy E, Bowen V, et al. Severe outcomes among patients with coronavirus disease 2019 (COVID-19) - United States, February 12-March 16, 2020. MMWR Morb Mortal Wkly Rep 2020; 69: 343-6.

16. Wang $Y$, Wang $Y$, Chen $Y$, Qin $Q$. Unique epidemiological and clinical features of the emerging. novel coronavirus pneumonia (COVID-19) implicate special control measures. J Med Virol 2020; DOI: https://doi.org/10.1002/jmv.25748.

17. Sanche $S$, Lin $Y T, X u C$, et al. High contagiousness and rapid spread of severe acute respiratory syndrome coronavirus 2 . Emerg Infect Dis 2020; DOI: https://doi.org/10.3201/eid2607. 200282.

18. Burke RM, Midgley CM, Dratch A, et al. Active monitoring of persons exposed to patients with confirmed COVID-19 - United States, January-February 2020. MMWR Morb Mortal Wkly Rep 2020; 69: 245-6.

19. Liu Y, Eggo RM, Kucharski AJ. Secondary attack rate and superspreading events for SARS-CoV-2. Lancet 2020; DOI: https://doi.org/10.1016/S0140-6736(20)30462-1.

20. Pastick KA, Okafor EC, Wang F, et al. Review: hydroxychloroquine and chloroquine for treatment of SARSCoV-2 (COVID-19). Open Forum Infect Dis 2020; DOI: https:// doi.org/10.1093/ofid/ofaa130.

21. Vincent $M J$, Bergeron E, Benjannet $S$, et al. Chloroquine is a potent inhibitor of SARS coronavirus infection and spread. Virol J 2005; DOI: https://doi.org/10.1186/1743-422X-2-69.

22. Yao $X$, Ye $F$, Zhang $M$, et al. In vitro antiviral activity and projection of optimized dosing design of hydroxychloroquine for the treatment of severe acute respiratory syndrome coronavirus 2 (SARS-CoV-2). Clin Infect Dis 2020; DOI: https://doi.org/10. 1093/cid/ciaa237.

23. Zhou D, Dai SM, Tong Q. COVID-19: a recommendation to examine the effect of hydroxychloroquine in preventing infection and progression. J Antimicrob Chemother 2020; DOI: https://doi. org/10.1093/jac/dkaa114.

24. Borba MG, de Almeida Val F, Sampaio VS, et al. Chloroquine diphosphate in two different dosages as adjunctive therapy of hospitalized patients with severe respiratory syndrome in the context of coronavirus (SARS-CoV-2) infection:pPreliminary safety results of a randomized, double-blinded, phase IIb clinical trial (CloroCovid-19 Study). medRxiv 2020; DOI: https://doi.org/ 10.1101/2020.04.07.20056424.

25. Lexi Drugs. Chloroquine. Hudson, OH: Lexi-Comp Inc.; 2020. Available from URL: https://online.lexi.com/lco/action/login (accessed April 2020).

26. McChesney EW. Animal toxicity and pharmacokinetics of hydroxychloroquine sulfate. Am J Med 1983; 75: 11-8. 
27. Lexi Drugs. Hydroxychloroquine. Hudson, OH: Lexi-Comp Inc.; 2016. Available from URL: https://online.lexi.com/lco/action/ login (accessed April 2020).

28. Tang W, Cao Z, Han $M$, et al. Hydroxychloroquine in patients with COVID-19: an open-label, randomized, controlled trial. medRxiv 2020; DOI: https://doi.org/10.1101/2020.04.10. 20060558.

29. Molina JM, Delaugerre C, Le Goff J, et al. No evidence of rapid antiviral clearance or clinical benefit with the combination of hydroxychloroquine and azithromycin in patients with severe COVID-19 infection. Med Mal Infect 2020; DOI: https://doi.org/ 10.1016/j.medmal.2020.03.006.

30. Wang $D, H u B, H u C$, et al. Clinical characteristics of 138 hospitalized patients with 2019 novel coronavirus-infected pneumonia in Wuhan, China. JAMA 2020; DOI: https://doi.org/ 10.1001/jama.2020.1585.

31. Huang C, Wang Y, Li X, et al. Clinical features of patients infected with 2019 novel coronavirus in Wuhan. China. Lancet 2020; 395: 497-506.

32. Mahevas $M$, Tran VT, Roumier $M$, et al. No evidence of clinical efficacy of hydroxychloroquine in patients hospitalized for COVID-19 infection with oxygen requirement: results of a study using routinely collected data to emulate a target trial. medRxiv 2020: DOI: https://doi.org/10.1101/2020.04.10. 20060699.

33. International Society of Nephrology. Kidney Disease: Improving Global Outcomes (KDIGO). KDIGO 2012 Clinical Practice Guideline for the Evaluation and Management of Chronic Kidney Disease; 2012. Kidney International Supplements January 2013. Available from URL: https://kdigo.org/wp-content/uploads/2017/ 02/KDIGO_2012_CKD_GL.pdf (accessed April 2020).

34. Organization of Teratology Information Specialists (OTIS). Mother to Baby Fact Sheet. Hydroxychloroquine. Available from URL: https://mothertobaby.org/fact-sheets/ hydroxychloroquine-pregnancy/pdf/. Published 2018. Accessed April 5, 2020 (accessed April 2020).
35. Centers for Disease Control and Prevention. Treatment of Malaria (Guidelines for Clinicians); 2019. Available from URL: https://www.cdc.gov/malaria/resources/pdf/clinicalguidance.pdf. (accessed April 2020).

36. World Health Organization. Global surveillance for human infection with coronavirus disease (COVID-19); 2020. Available from URL: https://www.who.int/publications-detail/ global-surveillance-for-human-infection-with-novel-coronavirus(2019-ncov) (accessed April 2020).

37. World Health Organization. COVID-19 Therapeutic Trial Synopsis; 2020. Available from URL: https://www.who.int/ blueprint/priority-diseases/key-action/COVID-19_Treatment_ Trial_Design_Master_Protocol_synopsis_Final_18022020.pdf (accessed April 2020).

38. Cheng MP, Papenburg J, Desjardins $M$, et al. Diagnostic testing for severe acute respiratory syndrome-related coronavirus-2: a narrative review. Ann Intern Med 2020; DOI: https://doi.org/10. 7326/M20-1301.

39. Centers for Disease Control and Prevention. Information for clinicians on investigational therapeutics for patients with COVID-19; 2020. Available from URL: https://www.cdc.gov/ coronavirus/2019-ncov/hcp/therapeutic-options.html (accessed April 2020).

40. Cheng MP, Lee TC, Tan DH, Murthy S. Generating randomized trial evidence to optimize treatment in the COVID-19 pandemic. CMAJ 2020; DOI: https://doi.org/10.1503/cmaj.200438.

41. Multicenter collaboration group of Department of Science and Technology of Guangdong Province and Health Commission of Guangdong Province for chloroquine in the treatment of novel coronavirus pneumonial. Expert consensus on chloroquine phosphate for the treatment of novel coronavirus pneumonia (Chinese). Zhonghua Jie He He Hu Xi Za Zhi 2020; 43: 185-8.

Publisher's Note Springer Nature remains neutral with regard to jurisdictional claims in published maps and institutional affiliations. 